\title{
Topological modes in relativistic hydrodynamics
}

\author{
Yan $\mathrm{Liu}^{1,2, *}$ and Ya-Wen Sun ${ }^{3,4, \dagger}$ \\ ${ }^{1}$ Center for Gravitational Physics, Department of Space Science, Beihang University, \\ Beijing 100191, China \\ ${ }^{2}$ Key Laboratory of Space Environment Monitoring and Information Processing, \\ Ministry of Industry and Information Technology, Beijing 100191, China \\ ${ }^{3}$ School of physics and CAS Center for Excellence in Topological Quantum Computation, \\ University of Chinese Academy of Sciences, Beijing 100049, China \\ ${ }^{4}$ Kavli Institute for Theoretical Sciences, University of Chinese Academy of Sciences, \\ Beijing 100049, China
}

(Received 28 April 2020; accepted 3 February 2021; published 23 February 2021)

\begin{abstract}
We show that gapless modes in relativistic hydrodynamics could become topologically nontrivial by weakly breaking the conservation of energy momentum tensor in a specific way. This system has topological semimetal-like crossing nodes in the spectrum of hydrodynamic modes that require the protection of a special combination of translational and boost symmetries in two spatial directions. We confirm the nontrivial topology from the existence of an undetermined Berry phase. These energy momentum nonconservation terms could naturally be produced by an external gravitational field that comes from a reference frame change from the original inertial frame, i.e., by fictitious forces in a noninertial reference frame. This noninertial frame is the rest frame of an accelerating observer moving along a trajectory of a helix. This suggests that topologically trivial modes could become nontrivial by being observed in a special noninertial reference frame, and this fact could be verified in laboratories, in principle. Finally, we propose a holographic realization of this system.
\end{abstract}

DOI: 10.1103/PhysRevD.103.044044

\section{INTRODUCTION}

Hydrodynamics is the universal low energy theory for systems close to local thermal equilibrium at a long distance and time. It could describe a variety of physical systems ranging from matter at large scales in the Universe, the quarkgluon plasma [1], to Weyl semimetals [2,3] and graphenes [4] in the laboratory. At small momentum and frequency, perturbations of a hydrodynamic system close to the equilibrium would produce propagating as well as diffusive modes [5]. These modes are gapless, whose poles are at $\omega=\mathbf{k}=0$, which reflects the fact that energy momentum is conserved.

During the last decade, topologically nontrivial quantum states have been discovered in condensed matter physics $[6,7]$. Later, it has been found that many classical systems have nontrivial topological states too, including topological optical/sound systems (see, e.g., [8-10] and references therein), which have also been observed experimentally.

\footnotetext{
yanliu@buaa.edu.cn

yawen.sun@ucas.ac.cn
}

Published by the American Physical Society under the terms of the Creative Commons Attribution 4.0 International license. Further distribution of this work must maintain attribution to the author(s) and the published article's title, journal citation, and DOI. Funded by SCOAP ${ }^{3}$.
It raises the question if the gapless modes in relativistic hydrodynamics could also become topologically nontrivial under certain conditions. In this paper, we start from the relativistic hydrodynamics and show that after weakly breaking conservation of energy momentum, hydrodynamic modes could become topological semimetal-like nontrivial states that require the protection of a special spacetime symmetry, and interestingly, these nonconservation terms for the energy momentum tensor could come from a noninertial reference frame of an accelerating observer moving along a helix.

\section{EFFECTIVE HAMILTONIAN AND SPECTRUM IN RELATIVISTIC HYDRODYNAMICS}

We focus on the simplest hydrodynamic systems with no internal charges, whose only conserved quantity is the energy momentum tensor that satisfies $\partial_{\mu} T^{\mu \nu}=0$. Up to the first order derivative, the constitutive equation for the energy momentum tensor in the Landau frame is

$$
\begin{aligned}
T^{\mu \nu}= & \epsilon u^{\mu} u^{\nu}+P \Delta^{\mu \nu}-\eta \Delta^{\mu \alpha} \Delta^{\nu \beta}\left(\partial_{\alpha} u_{\beta}+\partial_{\beta} u_{\alpha}\right. \\
& \left.-\frac{2}{3} \eta_{\alpha \beta} \partial_{\sigma} u^{\sigma}\right)-\zeta \Delta^{\mu \nu} \partial_{\alpha} u^{\alpha}+\mathcal{O}\left(\partial^{2}\right),
\end{aligned}
$$


where $\Delta_{\mu \nu}=\eta_{\mu \nu}+u_{\mu} u_{\nu}, \epsilon, P$ are the energy densities, and pressure and $\eta, \zeta$ are the shear and bulk viscosities.

With small perturbations away from the equilibrium, the system would respond to the perturbations and develop hydrodynamic modes. There are four eigenmodes of the system. Two of them are the sound modes propagating in the direction of $\mathbf{k}=\left(k_{x}, k_{y}, k_{z}\right)$ with the dispersion relation $\omega= \pm v_{s} k-i \Gamma_{s} k^{2}$, where $v_{s}=\sqrt{\frac{\partial P}{\partial \epsilon}}$ and $\Gamma_{s}=\left(\frac{4}{3} \eta+\zeta\right) /$ $(\epsilon+P)$. The other two are transverse modes with $\omega=-i \frac{\eta}{\epsilon+P} k^{2}$. To the first order in $k$, dissipative terms disappear, and the spectrums of the four modes are real, which cross each other at $\omega=\mathbf{k}=0$. This spectrum looks similar to the spectrum of Dirac semimetals, except that we have two extra flat bands here.

To change the spectrum to a topological semimetal-like one, we need to add nonconservation terms of $T^{\mu \nu}$ into the conservation equations. As a first step, we develop the notion of an effective Hamiltonian in hydrodynamics. Substituting the constitutive equations for the perturbations $\delta T^{\mu \nu}$ into $\partial_{\mu} \delta T^{\mu \nu}=0$, we could rewrite the equations into the form,

$$
i \partial_{t} \Psi=H \Psi,
$$

where we have defined

$$
\Psi=\left(\begin{array}{c}
\delta \epsilon \\
\delta \pi^{x} \\
\delta \pi^{y} \\
\delta \pi^{z}
\end{array}\right), \quad H=\left(\begin{array}{cccc}
0 & k_{x} & k_{y} & k_{z} \\
k_{x} v_{s}^{2} & 0 & 0 & 0 \\
k_{y} v_{s}^{2} & 0 & 0 & 0 \\
k_{z} v_{s}^{2} & 0 & 0 & 0
\end{array}\right),
$$

at leading order in $k$, i.e., omitting dissipative terms at $\mathcal{O}\left(k^{2}\right)$.

In this way, in analogy to the electronic systems [11] we have defined an effective Hamiltonian matrix $H$, whose eigenvalues give the spectrum of hydrodynamic modes [12]. The four eigenvalues of the matrix Hamiltonian above give the sound modes $\omega= \pm v_{s} \sqrt{k_{x}^{2}+k_{y}^{2}+k_{z}^{2}}$ and double copies of transverse modes $\omega=0$. The form (2) is the "free" Hamiltonian matrix for a conserved energy momentum tensor.

\section{TOPOLOGICALLY NONTRIVIAL MODES}

To deform the spectrum of the hydrodynamic modes, we introduce nonconservation terms for the energy momentum tensor and make sure that the nonconservation terms are small enough to stay within the hydrodynamic limit. The nonconservation of energy and momentum could come from a certain external system, which couples to the hydrodynamic system under study. At this stage, we assume that the constitutive equations for $T^{\mu \nu}$ do not get modified, and later, we will take the modifications into account and show that the spectrum does not change up to a rescaling of parameters.

We take a 4D hydrodynamic system and introduce nonconservation terms for $T^{\mu \nu}$ as follows:

$$
\begin{aligned}
& \partial_{\mu} \delta T^{\mu t}=m \delta T^{t x}, \quad \partial_{\mu} \delta T^{\mu x}=-m v_{s}^{2} \delta T^{t t}, \\
& \partial_{\mu} \delta T^{\mu y}=b v_{s} \delta T^{t z}, \quad \partial_{\mu} \delta T^{\mu z}=-b v_{s} \delta T^{t y},
\end{aligned}
$$

where $m$ terms gap the spectrum, while $b$ terms change the momentum position of the crossing nodes in the spectrum, and we assume $\mathcal{O}\left(k^{2}\right)<\mathcal{O}(m, b) \lesssim \mathcal{O}(k)$.

Physically, (3) states that energy (momentum in the $x$ direction) is not conserved, whose nonconservation is proportional to the momentum in the $x$ direction (energy). Later, we will show that these seemingly ad hoc nonconservation terms naturally arise from the observation of an accelerating observer moving in a helix, which could be tested in experiments, in principle.

After substituting the fluctuations of constitutive equation into (3), we obtain

$$
i \partial_{t} \Psi=H \Psi,
$$

where $\Psi=\left(\delta \epsilon, \delta \pi^{x}, \delta \pi^{y}, \delta \pi^{z}\right)^{T}$ and

$$
H=\left(\begin{array}{cccc}
0 & k_{x}+i m & k_{y} & k_{z} \\
\left(k_{x}-i m\right) v_{s}^{2} & 0 & 0 & 0 \\
k_{y} v_{s}^{2} & 0 & 0 & i b v_{s} \\
k_{z} v_{s}^{2} & 0 & -i b v_{s} & 0
\end{array}\right) .
$$

$H$ is similar to a Hermitian matrix as could be seen by redefining $\delta \epsilon \rightarrow \frac{1}{v_{s}} \delta \epsilon$. Thus, this effective $H$ has real eigenvalues, and the factor $v_{s}$ could be ignored, which could be taken back by an inverse transformation when necessary.

The spectrum of the hydrodynamic modes for (5),

$$
\omega= \pm \frac{1}{\sqrt{2}} \sqrt{b^{2}+k^{2}+m^{2} \pm \sqrt{\left(k_{x}^{2}+m^{2}-b^{2}\right)^{2}+\left(k_{y}^{2}+k_{z}^{2}\right)^{2}+2\left(k_{y}^{2}+k_{z}^{2}\right)\left(k_{x}^{2}+m^{2}+b^{2}\right)}},
$$

where $k=\sqrt{k_{x}^{2}+k_{y}^{2}+k_{z}^{2}}$. 


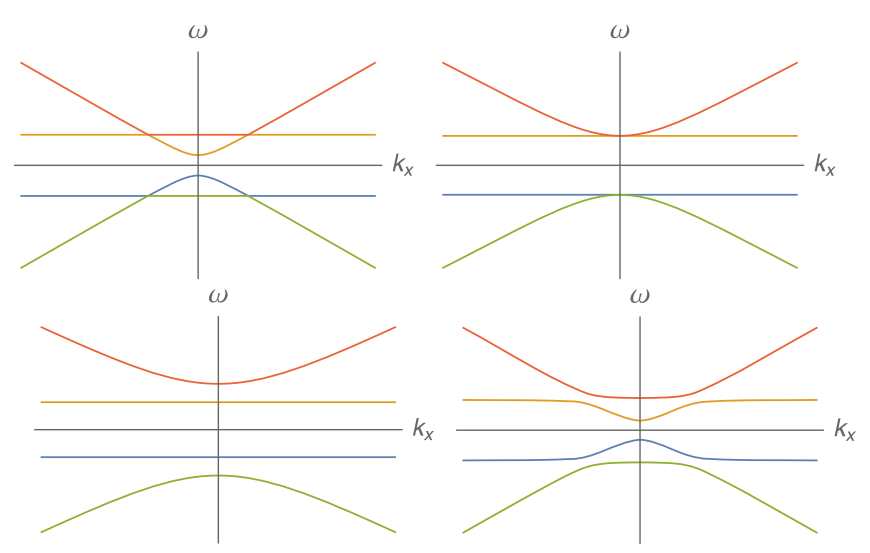

FIG. 1. The spectrum of the modified hydrodynamics with dynamical equation (3). From left up to right down: the first three plots are for $m<b, m=b$, and $m>b$, respectively, and $k_{y}=k_{z}=0$. The fourth plot is for $k_{y}>0, k_{z}=0$, and $m<b$. The distance between two flat bands is determined by the value of $b$, while the gap between two curved bands is determined by the parameter $m$.

Figure 1 shows this spectrum as a function of $k_{x}$ for $k_{y}=k_{z}=0$ in three different situations: $m<b, m=b$ and $m>b$ as well as for $k_{y}>0, k_{z}=0$ at $m<b$. The effect of $m$ terms is to gap the two sound modes. The effect of $b$ terms is to lift and lower the two transverse flat bands to symmetric positions of opposite sides of the $k$ axis. In this way, the modes have band crossings at nonzero values of $k$ for $m<b$.

From Fig. 1, we could see that for $m<b$, there are four band crossing nodes at $k_{y}=k_{z}=0$ while $k_{x} \neq 0$, and for these nodes, $\omega \neq 0$. These four nodes are still points in the expanded space of $\omega, k_{x}, k_{y}$, and $k_{z}$ as can be seen from the fourth plot in Fig. 1. For $m=b>0$, the system becomes critical with two nodes, and for $m>b$, the system becomes gapped again. This behavior is qualitatively similar to the topological phase transition of a topological semimetal [13].

The $m$ terms in (5) do not gap the four band crossing nodes in the $m<b$ case; however, if we have extra $m$ terms in the $y$ or $z$ directions, the gaps will open no matter how small the $y$ or $z$ mass parameters are. The spectrum in this case looks the same as the bottom right one in Fig. 1. In this situation, there are no crossing nodes anymore. This means that the nodes should be topologically nontrivial under the protection of symmetries that forbid the $m$ terms in the $y$ and $z$ directions. We will see later that the symmetry needed here is a special combination of translational and boost symmetry in $y$ and $z$ directions. In this sense, the system (3) experiences a symmetry protected topological phase transition that happens at the critical point $m=b$.

Note that for the hydrodynamical modes, $\omega(k=0)$ is not zero anymore due to the nonconservation of energy; i.e., energy is constantly pumped into or out of the system. These crossing nodes at $m<b$ are dissipative when order $k^{2}$ terms are taken into account. This is different from the $\omega=k=0$ nodes which are real poles in hydrodynamics with unbroken translational symmetries.

The new $m$ and $b$ terms above are not dissipative so they only change the shape of the spectrum while do not introduce any imaginary parts in the dispersion relation. In contrast, momentum dissipation terms in, e.g., [14-16] are dissipative terms.

\section{ORIGIN FOR NONCONSERVATION TERMS OF $T^{\mu \nu}$}

The simplest way to have the nonconservation terms of $T^{\mu \nu}$ in (3) is to introduce an external rank two symmetric tensor field. A natural possibility is for this external field to be a gravitational field $h_{\mu \nu}$ [17]. Then, the whole metric field is $g_{\mu \nu}=\eta_{\mu \nu}+h_{\mu \nu}$, and the energy momentum tensor is conserved as $\nabla_{\mu} T^{\mu \nu}=0$ in the new spacetime so that $\partial_{\mu} T^{\mu \nu}=0$ does not hold anymore. Expanding this equation in $h_{\mu \nu}$, we get

$\partial_{\mu} \delta T^{\mu \nu}=-\frac{1}{2} \partial_{\alpha} h \delta T^{\alpha \nu}-\frac{1}{2} \eta^{\nu \beta}\left(2 \partial_{\mu} h_{\alpha \beta}-\partial_{\beta} h_{\mu \alpha}\right) \delta T^{\mu \alpha}$.

Again, we have assumed that $\mathcal{O}\left(h_{\mu \nu}\right) \sim \mathcal{O}(k)$ and only kept leading order in $k$ terms [18]. To get the exact $m$ and $b$ terms in the effective Hamiltonian (5), there are infinite many choices for $h_{\mu \nu}$, and the simplest choice is for $h_{\mu \nu}$ to be [19]

$h_{t t}=h_{x x}=m x, \quad h_{t x}=h_{x t}=\frac{1}{2} m t\left(v_{s}^{2}+1\right)$,

$h_{t y}=h_{y t}=-\frac{1}{2} b v_{s} z, \quad h_{t z}=h_{z t}=\frac{1}{2} b v_{s} y$.

This graviton field $h_{\mu \nu}$ (7) could come from sources of massive matter, and more interestingly, it could also come from a reference frame transformation from the flat Minkowski metric generated by $\tilde{x}_{\mu}=x_{\mu}+\xi_{\mu}$ with [20],

$$
\xi_{\mu}=\left(\frac{m x t}{2}, \frac{m x^{2}}{4}+\frac{m t^{2}}{4} v_{s}^{2},-\frac{b}{4} v_{s} z t, \frac{b}{4} v_{s} y t\right) .
$$

This is an intriguing result as usually a nontrivial gravitational field could not be transformed to a flat spacetime globally but only locally. It could be checked that this new metric field has all the components of the Riemann tensor vanishing at leading order, thus could be transformed to the flat spacetime. Though equivalent to a flat spacetime, $h_{\mu \nu}$ could still be viewed as a nontrivial gravitational field according to the equivalence principle. This $h_{\mu \nu}$ denotes a noninertial reference frame. This result suggests that in a specific noninertial frame, we could observe hydrodynamic modes that are topologically protected even when they are 
topologically trivial in the original inertial frame. This could be tested in laboratories, in principle.

Note that with a nonzero $h_{\mu \nu}$, the constitutive equations for $T_{\mu \nu}$ could also be written into a covariant form thus leading to extra terms compared to the original constitutive equations. However, it can be explicitly checked that these extra terms do not change the spectrum (3) at all or do not change the spectrum up to a rescaling of parameters $m, b$, and $v_{s}$ depending on whether the fluid is resting in the original inertial frame or is accelerating together with the accelerating observer; i.e., the new spectrum could be obtained by substituting the rescaling relation above to the spectrum (3), and this does not mean that the real speed of sound changes. More details could be found in the Supplemental Material [21].

\section{THE EXACT NONINERTIAL REFERENCE FRAME}

We could work out the exact reference frame from the infinitesimal transformation (8). A rest observer in the new frame $\tilde{x}^{\mu}$ has $d \tilde{x}^{i}=0$ for $i=1,2,3$. From this, we could obtain $d \tilde{t}=d t, d x=-\frac{m v_{s}^{2} t d t}{2}, d y=\frac{b v_{s} z d t}{4}$, and $d z=-\frac{b v_{s} y d t}{4}$ at order $O(m)$. From the last two equations above, we have $y=R_{0} \cos \frac{b v_{s}}{4} t$ and $z=-R_{0} \sin \frac{b v_{s}}{4} t$ with appropriate choice for $t=0$ and a constant radius $R_{0}$. These together confirm that the rest observer of the new noninertial frame is in fact an accelerating observer in the original inertial frame who has a constant acceleration $a=-\frac{m v_{s}^{2}}{2}$ in the $x$ direction and a constant angular velocity $\omega_{x}=\frac{b v_{s}}{4}$ in the $y$ and $z$ plane. As $m$ and $b$ are small parameters, the observer is moving in the nonrelativistic limit, which is consistent with $d \tilde{t}=d t$. Note that the fluid is still a relativistic one though moving collectively nonrelativisitically.

Thus, the topological modes are those observed by an accelerating observer moving together with the fluid along a helix in the nonrelativistic limit as shown in Fig. 2, which makes it, in principle, a realizable setting for experimental tests of this system. Physically, the nonconservation terms for $T^{\mu \nu}$ could be thought of as coming from the fictitious force, including the Coriolis force, the centrifugal force as well as the inertial force associated with the $x$ direction constant acceleration. The nontrivial topological nodes

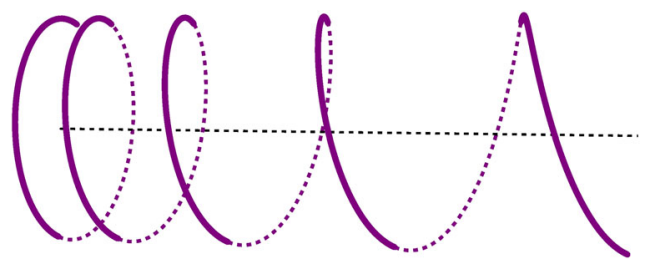

FIG. 2. The helix trajectory of the accelerating observer and the fluid in the laboratory frame. The observer/fluid has both a constant acceleration and a constant angular velocity in the $x$ direction. could also be viewed as coming from these fictitious effects. Now we have shown that the seemingly ad hoc nonconservation terms of $T^{\mu \nu}$ could in fact be generated from a very natural noninertial reference frame.

Now we could work out the symmetry of the system (3) as the isometry of the metric $g_{\mu \nu}=\eta_{\mu \nu}+h_{\mu \nu}$, i.e., coordinate transformations that leave $g_{\mu \nu}$ unchanged. The symmetry could be viewed as the Lie transformation of the Poincare symmetry generated by the vector (8). Among the ten generators of this new isometry [22], two of them are responsible for forbidding $m$ terms in the $y$ and $z$ directions and protecting the nontrivial topological states, which are generated by $x^{\mu} \rightarrow x^{\mu}+\epsilon^{\mu}$, where $\epsilon^{\mu}=a_{y} \chi_{y}+$ $a_{z} \chi_{z}$ with $\chi_{y}=\left(-\frac{b z v_{s}}{4}, 0,1,-\frac{b t v_{s}}{4}\right), \chi_{z}=\left(\frac{b y v_{s}}{4}, 0, \frac{b y v_{s}}{4}, 1\right)$ and $a_{y}, a_{z}$ two infinitesimal constants. $\chi_{y}$ (or $\chi_{z}$ ) is a special combination of the $y$ (or $z$ ) direction translational symmetry and the boost symmetry of the $t-y$ (or $t-z)$ direction. Though this symmetry looks complicated, physically it only requires the covariant conservation of momentum in the $y$ and $z$ directions in the noninertial frame; i.e., there are no extra external forces in the $y$ and $z$ directions.

Finally, we mention another possible circumstance to have this nonzero $h_{\mu \nu}$, which could arise in analog gravity systems; i.e., certain materials could give rise to effective hydrodynamic equations as if there exists a nontrivial gravitational field.

\section{TOPOLOGICAL INVARIANT}

For systems protected by a certain symmetry, we could calculate the topological invariant at a high symmetric point in the momentum space, which is $k_{y}=k_{z}=0$ in this case. There is a charge conjugation symmetry for the solutions, and we could focus on the lower two nodes in the left top plot of Fig. 1. Here, as we are in zero effective residual spatial dimension, the calculation of the topological invariant is different from the Berry phase or Berry curvature for a nodal line or Weyl semimetals. For the left node at $k_{x}=$ $k_{1}$ in the left top plot of Fig. 1, the green solution at the left limit $k_{x} \rightarrow k_{1-}$ and the right limit $k_{x} \rightarrow k_{1+}$ are denoted as $\left|n_{1}\right\rangle$ and $\left|n_{2}\right\rangle$ separately. We could define a Berry phase between the two states $e^{-i \alpha}=\frac{\left\langle n_{1} \mid n_{2}\right\rangle}{\left\langle n_{1} \mid n_{2}\right\rangle \mid}$ to denote the topological invariant here. If the Berry phase is an undetermined one; i.e., $\left|n_{1}\right\rangle$ and $\left|n_{2}\right\rangle$ are orthogonal to each other, the system would be topologically nontrivial as the two states cannot be connected without passing through a singularity, which means the lower band and the upper band could not be separated by small perturbations.

In (3), $\left|n_{1}\right\rangle=\frac{1}{\sqrt{2}}(0,0,-i, 1)$ and $\left|n_{2}\right\rangle=\frac{1}{\sqrt{1+\frac{1}{v_{s}^{2}}}} \times$ $\left(-\frac{i \sqrt{m^{2}+k_{x}^{2}}}{v_{s}\left(m+i k_{x}\right)}, 1,0,0\right)$. Therefore, $\left\langle n_{1} \mid n_{2}\right\rangle=0$, which means that the Berry phase is undetermined. From the argument above, the two bands cannot be separated easily by a gap 
without going through a topological phase transition. Similar behavior of an undetermined Berry phase has also happened for the holographic nodal line semimetals $[23,24]$.

This result confirms that the four nodes in Fig. 1 are topologically nontrivial protected by a special combination of translational and boost symmetry in the $y$ and $z$ directions. At the same time, the Berry phase accumulated through the whole circle around this node would be trivial, indicating that it is indeed topologically trivial without the symmetry.

\section{TRANSPORT PROPERTIES}

We can follow the calculations in [5,25] to compute the heat transport for this system to uncover more observational effects. We obtain

$$
\begin{aligned}
& \kappa_{x x}\left(\omega, k_{x}\right)=-\frac{i \omega(\epsilon+P)}{T\left(\left(k_{x}^{2}+m^{2}\right) v_{s}^{2}+i \frac{\eta}{\epsilon+P} \omega k_{x}^{2}-\omega^{2}\right)}, \\
& \kappa_{y y}\left(\omega, k_{x}\right)=\kappa_{z z}\left(\omega, k_{x}\right)=-\frac{k_{x}^{2} \eta+i \omega(\epsilon+P)}{T\left(b^{2} v_{s}^{2}+\left(i \omega+\frac{\eta}{\epsilon+P} k_{x}^{2}\right)^{2}\right)}, \\
& \kappa_{y z}\left(\omega, k_{x}\right)=-\kappa_{z y}\left(\omega, k_{x}\right)=\frac{(\epsilon+P) b v_{s}}{T\left(b^{2} v_{s}^{2}+\left(i \omega+\frac{\eta}{\epsilon+P} k_{x}^{2}\right)^{2}\right)} .
\end{aligned}
$$

With the formulas above, when $m=b=0$, all diagonal components of the dc heat transport diverge. For generic $m$ and $b$, we have vanishing dc heat transport $\kappa_{x x}(0,0)$, $\kappa_{y y}(0,0)$ and $\kappa_{z z}(0,0)$ while $\kappa_{y z}(0,0)=-\kappa_{z y}(0,0)=$ $\frac{\epsilon+P}{T b v_{s}}$. These $m$ and $b$ terms eliminate the unphysical divergence of dc heat transports and lead to interesting vanishing dc heat transport behavior. An intuitive and physical reason for this system to be a dc thermal insulator could be seen from the spectrum, where there is an energy gap at $k=0$ in contrast to being gapless at $k=0$ for standard hydrodynamics, as the consequence of energy nonconservation. This is similar to the mechanism of finite dc conductivity obtained for cases with momentum dissipation.

\section{VIII. $\mathcal{O}\left(k^{2}\right)$ EFFECTS}

$\mathcal{O}\left(k^{2}\right)$ terms lead to dissipative effects and give rise to imaginary parts of frequency in the spectrum. The $\mathcal{O}\left(k^{2}\right)$ terms make the effective Hamiltonian matrix nonHermitian. Here, we still keep terms at $m \sim b$ order while not $m^{2} \sim b^{2}$ order assuming that $m \sim k^{2}$.

From the eigenvalues of Hamiltonian with $\mathcal{O}\left(k^{2}\right)$ effects included, we find that the real part has not changed while imaginary parts appear. At the four nodes, the imaginary parts are not zero indicating that the four nodes are dissipative in comparison to nondissipative nodes at $\omega=0$ in the usual hydrodynamics. The imaginary part for each of the band has a jump at the crossing nodes at $k_{y}=0$ in the $k_{x}$ axis; i.e., the imaginary parts of the same band are different at the left and right limits of the singular node. This behavior is similar to the behavior of the eigenstates when calculating the Berry phase and thus provides another piece of evidence of the existence of a symmetry protected topological singular node.

\section{WARD IDENTITIES AND HOLOGRAPHIC REALIZATION}

The physics of hydrodynamics has been studied extensively in holography for strongly coupled systems [26-28]. We aim to construct a holographic system possessing the same nonconservation equation of (3), thus providing an example of this system in the strongly coupled limit.

Holographically, we could also perform a coordinate transformation to get a noninertial frame version of the AdS/CFT correspondence, which has the metric $g^{\mu \nu}=$ $\eta_{\mu \nu}+h_{\mu \nu}$ at the boundary. This system should have the same spectrum of the hydrodynamic modes. As a first step for a confirmation, we need to show that our holographic system indeed has the nonconservation of (3). For this purpose, we will first obtain the Ward identities for $T^{\mu \nu}$ in the nonconserved hydrodynamic system from (3) and match these identities to those in the holographic noninertial frame system.

In the case that these nonconservation terms come from a gravitational field, we could start from the covariant conservation equation $\nabla_{\mu} T^{\mu \nu}=0$ and differentiate it with respect to $g_{\lambda \rho}$ to obtain the Ward identities in the momentum space of the boundary system. To the first order in $h_{\mu \nu}$, the Ward identities are

$$
\begin{aligned}
& k_{\mu} G^{\mu \nu, \lambda \rho}(k)+i\left[\Gamma^{(1) \mu}{ }_{\mu \alpha} G^{\alpha \nu, \lambda \rho}(k)+\Gamma_{\mu \alpha}^{(1) \nu} G^{\mu \alpha, \lambda \rho}(k)\right] \\
& \quad+\text { contact terms }=0
\end{aligned}
$$

where the explicit form of the contact terms is omitted. With nonzero $h_{\mu \nu}$, several components of $\Gamma_{\mu \alpha}^{(1) \nu}$ would be nonzero and contribute extra terms to the Ward identities of $k_{\mu} G^{\mu \nu, \lambda \rho}(k)+$ contact terms $=0$ in hydrodynamics systems with conserved $T^{\mu \nu}$ [29].

The Ward identities (9) could be reproduced from the holographic noninertial frame system, where we start from the usual anti-de Sitter (AdS) Schwartzchild black hole and perform coordinate transformations so that the boundary metric becomes $g_{\mu \nu}=\eta_{\mu \nu}+h_{\mu \nu}$.

We have checked that the holographic Ward identities match exactly to the hydrodynamic Ward identities (9). The details will be presented in [22], and the hydrodynamic modes and Green functions will be systematically studied in future work. 


\section{OUTLOOK}

One important application of the observation of topologically nontrivial hydrodynamics modes at finite frequency would be to enlarge the amplitudes at the crossing frequencies and momenta due to the doubling of modes, and this enlargement could be stable from perturbations under certain conditions.

The fact that an accelerating observer moving along a helix would see topological hydrodynamic modes of a hydrodynamic system moving in the same helix could, in principle, be checked in experiments, including probing the spectrum of the modes and measuring the featured transport properties of the system.

Finally, and most importantly, it is possible that systems other than hydrodynamic systems, e.g., electronic/photonic systems, would also become topologically nontrivial being observed in a certain noninertial frame [30], which would provide another way to obtain topologically nontrivial materials by mechanically accelerating the detector in a laboratory. This brings up a new interesting effect for accelerating observers in addition to the well-known Unruh effect [31].

\section{ACKNOWLEDGMENTS}

We would like to thank Matteo Baggioli, Rong-Gen Cai, Koenraad Schalm, Qing Zhang, anonymous referees, and especially Karl Landsteiner for useful discussions. This work is supported by the National Key R and D Program of China (Grant No. 2018FYA0305800). The work of Y. L. was also supported by the National Natural Science Foundation of China Grant No. 11875083. The work of Y. W. S. has also been partly supported by starting grants from University of Chinese Academy of Sciences and Chinese Academy of Sciences, and by the Key Research Program of Chinese Academy of Sciences (Grant No. XDPB08-1), the Strategic Priority Research Program of Chinese Academy of Sciences, Grant No. XDB28000000.
[1] J. Casalderrey-Solana, H. Liu, D. Mateos, K. Rajagopal, and U. A. Wiedemann, Gauge/String Duality, Hot $Q C D$ and Heavy Ion Collisions (Cambridge University Press, Cambridge, England, 2011).

[2] K. Landsteiner, Y. Liu, and Y. Sun, Negative magnetoresistivity in chiral fluids and holography, J. High Energy Phys. 03 (2015) 127.

[3] A. Lucas, R. A. Davison, and S. Sachdev, Hydrodynamic theory of thermoelectric transport and negative magnetoresistance in Weyl semimetals, Proc. Natl. Acad. Sci. U.S.A. 113, 9463 (2016).

[4] A. Lucas and K. C. Fong, Hydrodynamics of electrons in graphene, J. Phys. Condens. Matter 30, 053001 (2018).

[5] P. Kovtun, Lectures on hydrodynamic fluctuations in relativistic theories, J. Phys. A 45, 473001 (2012).

[6] N. P. Armitage, E. J. Mele, and A. Vishwanath, Weyl and Dirac semimetals in three dimensional solids, Rev. Mod. Phys. 90, 015001 (2018).

[7] E. Witten, Three lectures on topological phases of matter, Riv. Nuovo Cimento 39, 313 (2016).

[8] L. Lu, J. Joannopoulos, and M. Soljai, Topological photonics, Nat. Photonics 8, 821829 (2014).

[9] T. Ozawa, H. M. Price, A. Amo, N. Goldman et al., Topological photonics, Rev. Mod. Phys. 91, 015006 (2019).

[10] X. Zhang, M. Xiao, Y. Cheng, M.-H. Lu, and J. Christensen, Topological sound, Nat. Commun. 1, 97 (2018).

[11] S.-Q. Shen, Topological Insulators: Dirac Equation in Condensed Matter, Springer Series in Solid-State Sciences (Springer, Singapore, 2017), https://doi.org/10.1007/978981-10-4606-3.

[12] Note that this effective Hamiltonian is different from the hydrodynamic Hamiltonian, e.g., in Sec. 2.4 of Ref. [5] in the sense that this effective Hamiltonian matrix $\Psi^{T}(-k) H \Psi(k)$ gives the "kinetic" part of the Hamiltonian while the latter only counts in the potential part associated with the source terms.

[13] K. Landsteiner, Y. Liu, and Y. Sun, Quantum Phase Transition Between a Topological and a Trivial Semimetal from Holography, Phys. Rev. Lett. 116, 081602 (2016).

[14] S. A. Hartnoll, P. K. Kovtun, M. Muller, and S. Sachdev, Theory of the Nernst effect near quantum phase transitions in condensed matter, and in dyonic black holes, Phys. Rev. B 76, 144502 (2007).

[15] S. A. Hartnoll, A. Lucas, and S. Sachdev, Holographic quantum matter, arXiv:1612.07324.

[16] S. Grozdanov, A. Lucas, and N. Poovuttikul, Holography and hydrodynamics with weakly broken symmetries, Phys. Rev. D 99, 086012 (2019).

[17] Another possibility for $f_{\mu \nu}$ could be found in Supplemental Material.

[18] We assume that we are working in a large but finite volume of spacetime and $m, b$ are so small that $m x, b y, b z, m t \ll 1$ in the finite volume. We ignore the boundary effects as the volume is large.

[19] Another general set of choices is to choose $h_{\mu \nu}$ to be $h_{t t}=$ $h_{x x}=m x, h_{t x}=c m t, h_{t y}=-b v_{s} z / 2, h_{t z}=b v_{s} y / 2, h_{x y}=$ $-m y, h_{x z}=e m z$, where the two constants $c$ and $e$ should satisfy $1-2 c+(1-2 e) v_{s}^{2}=-2 v_{s}^{2}$.

[20] The set of graviton fields in Ref. [19] could also be obtained from a similar coordinate transformation from the flat spacetime.

[21] See Supplemental Material at http://link.aps.org/ supplemental/10.1103/PhysRevD.103.044044 for another possible origin for $f_{\mu \nu}$ and the modification due to the change in the constitutive equations. 
[22] Y. Liu and Y. W. Sun, Topological hydrodynamic modes and holography, arXiv:2005.02850.

[23] Y. Liu and Y. Sun, Topological invariants for holographic semimetals, J. High Energy Phys. 10 (2018) 189.

[24] K. Landsteiner, Y. Liu, and Y. Sun, Holographic topological semimetals, Sci. China Phys. Mech. Astron. 63, 250001 (2020).

[25] R. A. Davison and B. Gouteraux, Momentum dissipation and effective theories of coherent and incoherent transport, J. High Energy Phys. 01 (2015) 039.

[26] P. Kovtun, D. T. Son, and A. O. Starinets, Viscosity in Strongly Interacting Quantum Field Theories from Black Hole Physics, Phys. Rev. Lett. 94, 111601 (2005).
[27] D. T. Son and A. O. Starinets, Viscosity, black holes, and quantum field theory, Annu. Rev. Nucl. Part. Sci. 57, 95 (2007).

[28] V. E. Hubeny, S. Minwalla, and M. Rangamani, The fluid/ gravity correspondence, arXiv:1107.5780.

[29] G. Policastro, D. T. Son, and A. O. Starinets, From AdS/CFT correspondence to hydrodynamics. 2. Sound waves, J. High Energy Phys. 12 (2002) 054.

[30] For fermionic systems, we have already had preliminary results showing that a non-inertial frame could also produce topologically nontrivial states from trivial states in the small momentum region.

[31] W. G. Unruh, Notes on black hole evaporation, Phys. Rev. D 14, 870 (1976). 